\title{
Identification of Germline Chimeric Chickens Produced by Transfer of Primordial Germ Cells Using a Hinai-dori-specific Microsatellite Marker
}

\author{
Kazuhiro Rikimaru ${ }^{1}$, Natsuki Ito ${ }^{2}$, Yoshiaki Nakamura, ${ }^{3,4}$, Daiki Takahashi ${ }^{1}$, \\ Manami Ono ${ }^{2}$, Megumi Komatsu ${ }^{1}$ and Kazuei Matsubara ${ }^{2}$ \\ ${ }^{1}$ Livestock Experiment Station, Akita Prefectural Agriculture Forestry and \\ Fisheries Research Center, Daisen 019-1701, Japan \\ ${ }^{2}$ Graduate School of Agriculture, Iwate University, Morioka, Iwate 020-8550, Japan \\ ${ }^{3}$ Interdisciplinary Graduate School of Science and Technology, Shinshu University, \\ Minamiminowa, Nagano 399-4598, Japan \\ ${ }^{4}$ Research Fellow of the Japan Society for the Promotion of Science, Japan
}

\begin{abstract}
The production of germline chimeric chickens by transfer of primordial germ cells (PGCs) is an effective technique for the preservation and regeneration of genetic resources in chickens. To date, the most widely used method to identify germline chimeric chickens is a testcross using differences in plumage color between the donor and recipient breeds. However, this method is time consuming and laborious. Therefore, simple high-precision molecular techniques for the identification of germline chimeric chickens need to be developed. In this study, we verified the practicability of using a Hinai-dori-specific microsatellite marker that was previously developed to identify germline chimeric chickens. A Hinai-dori-specific microsatellite marker ABR0633 can distinguish the Hinai-dori breed from White Leghorn (WL); thus, these two breeds were used as donor and recipient embryos, respectively, in this study. PGCs obtained from embryonic gonads of Hinai-dori were microinjected into either the subgerminal cavity or dorsal aorta of WL recipient embryos to produce germline chimeric chickens. A portion of both left and right testes was removed from the manipulated male chicks and was used for genotyping to verify the possibility of identifying germline chimeric chickens. Semen was then obtained from matured manipulated male chickens and was used for genotyping. Simultaneously, these chickens were crossed with Hinai-dori females by artificial insemination to produce offspring. No donor-derived Hinai-dori allele was detected in the testicular tissues from the manipulated male chicks. However, donor-derived Hinai-dori allele was detected in the semen from two manipulated male chickens $(2 / 2)$ that were produced by microinjection of Hinai-dori PGCs into the dorsal aorta. In the progeny tests, Hinai-dori PGC-derived offspring were obtained from these two chickens. Moreover, only the donor-derived Hinai-dori allele was detected in the offspring that were judged as the Hinai-dori breed by plumage color. Therefore, the offspring were confirmed as the Hinaidori breed both phenotypically and genotypically. We conclude that a Hinai-dori-specific microsatellite marker is suitable to identify germline chimeric chickens and the use of this method could reduce the time and labor needed for testcrosses.
\end{abstract}

Key words: chicken, germline chimeric chicken, Hinai-dori, microsatellite marker, primordial germ cells

\section{Introduction}

Recently, there was an outbreak of highly pathogenic avian influenza in Japan. Because of the death and slaughter disposal that occurred as a result of this infectious disease, there has been apprehension concerning the extinction of not only commercial breeds and lines, but also rare breeds of Japanese domestic fowl. An effective tech-

Received: June 1, 2011, Accepted: July 19, 2011

Released Online Advance Publication: August 25, 2011

Correspondence: Dr. K. Matsubara, Graduate school of Agriculture, Iwate University, Morioka, Iwate 020-8550, Japan.

(E-mail: kazuei@iwate-u.ac.jp) nique to avoid such extinction risks is the preservation of genetic poultry resources at the cellular level. In chicken, semen cryopreservation has been achieved; however, ova are not yet available for freezing in the same way. Techniques for the production of germline chimeric chickens by transfer of primordial germ cells (PGCs), which are progenitor cells of spermatozoa or ova, have been established using the reproduction technology research developed in birds (Petitte et al., 1990; Tajima et al., 1993). Naito et al. (1994) produced the first offspring from genetic resources in chicken preserved at the cellular level from germline chimeric chickens produced by transfer of frozen-thawed PGCs. This technique has been put to 
practical use for the preservation and regeneration of commercial breeding stocks and rare breeds. Kuwana et al. (2006) regenerated Kureko-dori, which was designated a Kumamoto prefectural natural treasure, via backcross of germline chimeric female chickens produced by transfer of frozen-thawed PGCs. Moreover, Nakamura et al. (2010a) regenerated Gifujidori fowl, which was designated a Japanese natural treasure, from cryopreserved PGCs via germline chimeric chickens produced by transfer of frozen-thawed PGCs. However, in these studies, there was a problem with low rates of donor-derived offspring. Nakamura et al. (2010b) enhanced the production rate of donor PGC-derived offspring in germline chimeric chickens, with the average rate of $99.5 \%$, by removing almost all endogenous PGCs in the recipient embryo. Furthermore, they produced only donor-derived offspring from germline chimeric chickens in which the recipient germlines were totally replaced by donor cells. These studies show that the production of germline chimeric chickens using PGCs is possible for the preservation and regeneration of genetic resources in chicken, and it is expected to be put to practical use.

Currently, testcrosse analysis is the most widely used method to identify germline chimeric chickens. Generally, differences in plumage color between the donor and the recipient breeds are used to identify germline chimeric chickens. Dominant White (I), a characteristic of White Leghorn (WL), is one of the major loci affecting plumage color. It is easily identified as a major genetic marker that distinguishes WL from colored (i) chicken breeds, and has been previously used to identify germline chimeric chickens (Han et al., 2002; Park et al., 2003; Naito and Kuwana, 2004; Kuwana et al., 2006; Mozdziak et al., 2006; Nakamura et al., 2010a, b). However, this method is time-consuming and laborious. Therefore, there is a need to develop simple high-precision molecular techniques for the identification of germline chimeric chickens.

Recently, some studies have used molecular methods to identify germline chimeric chickens. Naito et al. (2004) molecularly analyzed germline chimerism in the early embryo using a marker that distinguishes single nucleotide polymorphisms (SNPs) in the chicken mitochondrial DNA (mtDNA) D-loop. Hu et al. (2005) identified germline chimeric chickens using Amplified Fragment Length Polymorphism (AFLP) markers. Choi et al. (2007) detected donor-derived DNA from the semen of germline chimeric chickens using a 9-bp insertion in exon 10 of the Pre-Melanosomal Protein 17 (PMEL 17) gene that leads to a 3 -amino acid insertion in the PMEL 17 protein, which plays an important role in the synthesis of melanosomes. These studies show that it is possible to identify germline chimeric chickens using molecular methods.

The Hinai-dori, which is designated a Japanese natural treasure, is a chicken breed native to Akita Prefecture in Japan. Multiple microsatellite markers on the $\mathrm{Z}$ chromosome were linked to 1 allele of the Hinai-dori breed in the Livestock Experiment Station at the Akita Prefectural
Agriculture, Forestry, and Fisheries Research Center (ALES), which can distinguish Hinai-dori from other chicken breeds (Rikimaru and Takahashi, 2007). Moreover, these markers can also distinguish Hinai-jidori chicken (a commercial chicken), which is a cross between a Hinai-dori sire and a Rhode Island Red dam, from other chickens and eggs (Rikimaru and Takahashi, 2009, 2010).

In this study, we verified the practicability of a Hinaidori-specific microsatellite marker that was previously developed to identify germline chimeric chickens and confirmed the potential application of the Hinai-specific microsatellite marker to identify germline chimeric chickens.

\section{Materials and Methods}

\section{Fertilized Eggs and Animal Care}

Fertilized eggs from the Hinai-dori breed maintained at ALES and WL introduced from the National Livestock Breeding Center Okazaki Station (NLBC) were obtained by artificial insemination. All animal care and use in this study was conducted in accordance with the Guidelines for Proper Conduct of Animal Experiments (Science Council of Japan, 2006).

\section{Microsatellite Genotyping of Hinai-dori and WL}

Multiple microsatellite markers on the $\mathrm{Z}$ chromosome are linked to 1 allele in the Hinai-dori breed (Rikimaru and Takahashi, 2007). One of these Hinai-dori-specific microsatellite markers, ABR0633 (Forward primer, AGTATGTTATTGCCTGTGGC; Reverse primer, TTTGGGAGAAGGAATGTTGT) (Takahashi et al., 2005), can distinguish the Hinai-dori breed from WL that had previously been maintained at ALES. In this study, we verified whether it is possible to distinguish Hinai-dori from WL introduced from NLBC using the $A B R 0633$ marker.

Genomic DNA for polymerase chain reaction (PCR) amplification was extracted from the blood of matured Hinai-dori and WL. The PCR amplifications were performed in a $6-\mu \mathrm{L}$ reaction volume, which included 2.5 pmol of each primer, $200 \mu \mathrm{M}$ of each deoxynucleotide triphosphate, $1.2 \mathrm{mM} \mathrm{MgSO} 4,0.125$ units of KOD plus DNA polymerase (KOD-201; Toyobo, Tokyo, Japan), 1 $\times$ reaction buffer provided by the supplier, and $30 \mathrm{ng}$ genomic DNA, in a 96-well plate on the thermal cycler (GeneAmp System 9700; Perkin-Elmer, Foster City, CA, USA). The PCR was performed as follows: hot start at $94^{\circ} \mathrm{C}$ for $75 \mathrm{~s}$; followed by 10 cycles of $15 \mathrm{~s}$ at $94^{\circ} \mathrm{C}, 30 \mathrm{~s}$ at $60^{\circ} \mathrm{C}$, and $60 \mathrm{~s}$ at $68^{\circ} \mathrm{C}$, followed by 10 cycles using the same conditions except that the annealing temperature was $55^{\circ} \mathrm{C}$, and then 30 cycles with an annealing temperature of $50^{\circ} \mathrm{C}$, and a final elongation at $68^{\circ} \mathrm{C}$ for $9 \mathrm{~min}$. The PCR products were run with an internal size standard (Genescan 400HD ROX Size Standard; Perkin-Elmer) in an ABI PRISMTM 3130 DNA Sequencer (PerkinElmer). The sizes of fragments were analyzed using GeneMapper (Version 4.0; Perkin-Elmer). Alleles were designated according to PCR product size. 


\section{Production of Germline Chimeric Chickens}

(1) Collection of Embryonic Gonads and Preparation of Donor PGCs

The embryos of Hinai-dori breed were incubated in a forced air incubator (P-880(B); Showafuranki, Saitama, Japan) at $37.8^{\circ} \mathrm{C}$ and $55-60 \%$ relative humidity, with $90^{\circ}$ tilting once an hour. Freshly fertilized Hinai-dori eggs were incubated for 6 days to obtain the embryos at stage 29 (Hamburger and Hamilton, 1951). After disinfecting the eggshell with $70 \%$ alcohol, the eggs were broken and the contents were transferred to a laboratory dish (90 $\mathrm{mm})$. The embryo was washed with phosphate buffered saline (-) (PBS (-); Wako, Osaka, Japan) in the laboratory dish on a clean bench and the neck of the embryo was amputated. An incision was made in the abdomen of the embryo using tweezers and $27 \mathrm{G}$ hypodermic needle (3/4 inch; TERUMO, Tokyo, Japan) connected to a 1 - $m L$ syringe (TERUMO). After removing the heart, stomach, intestine, etc, the embryonic gonad was removed with the mesonephros under a stereoscopic microscope (SZK12: OLYMPUS, Tokyo, Japan). The embryonic gonad was separated from the mesonephros using a set of two $27 \mathrm{G}$ hypodermic needles connected to a $1-\mathrm{mL}$ syringe under a stereoscopic microscope. The embryonic gonad was placed into $100 \mu \mathrm{L}$ of PBS $(-)$ in a $1.5-\mathrm{mL}$ microfuge tube (BM, Equipment, Tokyo, Japan). After centrifugation at $2,000 \times \mathrm{g}$ for $4 \mathrm{~min}$ at room temperature, the PBS (-) was removed. Trypsin-EDTA $(0.25 \%$ Trypsin, 1 mM EDTA 4Na; GIBCO, Invitrogen, Carlsbad, CA, USA) $(100 \mu \mathrm{L})$ was added to the tube and the gonads were incubated at $37^{\circ} \mathrm{C}$ for $2 \mathrm{~min}$ in the tube. The gonad cells were dissociated by pipetting, and $1 \mathrm{~mL}$ of Dulbecco's Modified Eagle Medium (DMEM; Sigma-Aldrich, St. Louis, Missouri, USA) containing chick serum (10\%) and penicillin/streptomycin (penicillin concentration: $10,000 \mathrm{IU} / \mathrm{mL}$, streptomycin concentration: $10 \mathrm{mg} / \mathrm{mL}$ ) was added to the tube to stop the reaction. After centrifugation at $2,000 \times \mathrm{g}$ for $4 \mathrm{~min}$ at room temperature, the supernatant was removed. Trypsin-EDTA was removed by rinsing twice with the above-mentioned solution.

(2) Preparation of Recipient Embryos and Transfer of PGCs

The Hinai-dori gonadal-drived PGCs (gPGC) were microinjected into either the subgerminal cavity of newly laid eggs (stage X: Eyal-Giladi and Kochav, 1976) or the dorsal aorta of WL recipient embryos at stages $12-13$ to produce germline chimeric chickens. The embryos that were microinjected with the Hinai-dori PGCs into the subgerminal cavity at stage $\mathrm{X}$ were cultured ex vivo (Perry, 1988; Naito et al., 1990).

Fertilized WL eggs were broken and the yolks were transferred to small host eggshells prepared from freshly laid eggs by cutting off the sharp ends of the shells (diameter of the hole, $33 \mathrm{~mm}$ ). The shells were filled with thin albumen obtained from other freshly laid eggs. Ultraviolet radiation (UV) (wavelength: $360 \mathrm{~nm}$ ) was applied to the WL recipient blastoderm from a distance of $1 \mathrm{~cm}$ for 60 seconds at a strength of $1600 \mathrm{~mW} / \mathrm{cm}^{2}$ to remove the endogenous PGCs (UV-400: Keyence, Osaka, Japan). The gPGCs were morphologically distinguished from the somatic cells under an inverted microscope (TS1B; Nikon, Tokyo, Japan) (Tajima et al., 1998). Under a microscope, $50-100$ gPGCs were collected using a fine glass micropipette (inner diameter, $0.69 \mathrm{~mm}$; outer diameter, $1.19 \mathrm{~mm}$; beveled tip $\left(20^{\circ}\right)$ to an outer diameter of $50 \mu \mathrm{m}$; Drummond Scientific, Broomall, PA, USA), and were microinjected into the WL recipient embryo. The host eggshells were sealed with cling film secured by plastic rings and rubber bands. The embryos were incubated in a forced air incubator at $39.0^{\circ} \mathrm{C}$ and $55-60 \%$ relative humidity with $90^{\circ}$ tilting 16 times an hour for $48-52 \mathrm{~h}$. When the manipulated embryos reached stage $16-17$, the embryos and yolks were transferred to large host eggshells made by drilling off the blunt ends of freshly laid doubleyolk eggs (diameter of hole, $38 \mathrm{~mm}$ ). After removing 3$5 \mathrm{~mL}$ of albumen, the large host eggshells were sealed with cling film. The embryos were incubated in a forced air incubator at $38.5^{\circ} \mathrm{C}$ and $50-60 \%$ relative humidity with $30^{\circ}$ tilting twice an hour for about 18 days. After the manipulated embryos were incubated for 18-20 days, tilting was stopped, and they were transferred to a hatching tray. A minimal hole was made in the cling film to allow the manipulated embryos to respire, and a laboratory dish was put on the film to prevent the manipulated embryos from drying. The cling film was taken off and only the laboratory dish was put on the large host eggshells just before hatching. When the chicks hatched without help on the 21st incubation day, the chicks were defined as hatching.

When Hinai-dori PGCs were transferred into bloodstream of recipient embryos at stages 12-13, a windowopen method was used. The WL embryos were incubated in a forced air incubator at $39.0^{\circ} \mathrm{C}$ and $55-60 \%$ relative humidity with $90^{\circ}$ tilting 1 time an hour for $48-52 \mathrm{~h}$. A window (diameter, $1.0-1.5 \mathrm{~cm}$ ) was opened at the sharp end of eggshell after making a small hole at the blunt end, which made manipulation of the embryos easy because the air chamber at the sharp end of the eggshell was crushed and the position of the embryos fell. Blood $(3.5-6 \mu \mathrm{L})$ was picked up from the dorsal aorta of the recipient embryos according to the method of Naito et al. (1994b). Under a microscope, 50-100 gPGCs were collected using a fine glass micropipette (inner diameter, $0.69 \mathrm{~mm}$; outer diameter, $1.19 \mathrm{~mm}$; beveled tip $\left(20^{\circ}\right)$ to an outer diameter of $50 \mu \mathrm{m}$; Drummond Scientific), and were microinjected into the dorsal aorta of WL recipient embryos through the window in the eggshell under a stereoscopic microscope. After removing $2-3 \mathrm{~mL}$ of albumen, the window in the eggshell was sealed with cling film, and the eggs were cultured in a forced air incubator at $38.5^{\circ} \mathrm{C}$ and $50-60 \%$ relative humidity with $30^{\circ}$ tilting 2 times an hour for approximately 18 days. After the manipulated embryos were incubated for 18-20 days, the tilting was stopped and they were transferred to a hatching tray. When the chicks 
hatched without help on the 21st incubation day, the chicks were defined as hatching.

Preparation of DNA from Testes and Semen

(1) DNA extraction from testes

At 5-weeks old, a portion of both left and right testes of the manipulated male chicks was removed according to the caponization method of Rikimaru et al. (2011). Briefly, the chicks were fastened to a clean wooden work surface on a table, and an incision was made using a knife between the last 2 ribs, which were widened using a spreader. About $1 / 3$ of the testis was removed using caponizing forceps (K-2; Natsume Seisakusho, Tokyo, Japan), and then a part of the other testis was removed in a similar manner. After removing the spreader, the wound was disinfected with $70 \%$ ethanol.

The collected testicular tissues were placed into a 2.0$\mathrm{mL}$ microfuge tube (BM, Equipment), to which, $750 \mu \mathrm{L}$ of a solution (10 mM Tris-HCL ( $\mathrm{pH} 8.0), 1 \mathrm{mM}$ EDTA (pH 8.0), $150 \mathrm{mM} \mathrm{NaCl}$, and $1 \% \mathrm{SDS}$ ) and $5 \mu \mathrm{L}$ of proteinase $\mathrm{K}$ (Wako) was added. The tubes were then shaken sufficiently and heated at $50^{\circ} \mathrm{C}$. After dissolving the testicular tissues, the tube contents were preserved at $4{ }^{\circ} \mathrm{C}$. Following the addition of an equal volume of phenol to the nucleic acid solution, the tubes were centrifuged at $2,000 \times \mathrm{g}$ for $5 \mathrm{~min}$. The aqueous layer at the top of the tube was transferred to a new tube, and the abovementioned procedure was repeated until the layer at the interface was removed. After the interface layer was gone, an equal volume of PCI (phenol: chloroform: isoamyl alcohol, 25: 24: 1, Wako) was added to the tube containing the nucleic acid solution. The tube was centrifuged at $2,000 \times \mathrm{g}$ for $5 \mathrm{~min}$, and then the aqueous layer at the top was transferred to a new tube. The procedure was repeated till the layer at the interface was removed. Following the addition of $1 / 10$ volume of $3 \mathrm{M}$ sodium acetate to the nucleic acid solution, $2.5 \times$ volumes of isopropyl alcohol were added, and the mixture was stirred. Then, the tube contents were centrifuged at $14,000 \times \mathrm{g}$ for $20 \mathrm{~min}$, and the supernatant was carefully removed. After $1 \mathrm{~mL}$ of $70 \%$ ethanol was added to the tube, it was centrifuged at $14,000 \times \mathrm{g}$ for $20 \mathrm{~min}$, and the supernatant was removed again. The pellet in the tube was dried completely and was dissolved in sterile deionized water.

(2) DNA extraction from semen

The manipulated male chicks with a part of their testes removed were raised to maturity, and semen was collected using the massage method. The collected semen was dropped on an FTA card (WB120205; GE Healthcare, Buckinghamshire, UK) and was dried at room temperature. Five disks were punched from the card using a Harris Micro Punch, which has a diameter of $1.2 \mathrm{~mm}$, and were put into a $0.2-\mathrm{mL}$ microfuge tube. After the addition of $100 \mu \mathrm{L}$ of FTA Purification Reagent, the disks were stirred and incubated for $20 \mathrm{~min}$. Following the removal of the supernatant, $100 \mu \mathrm{L}$ of DNAzol BD was added to the tube, and then stirred and incubated for $20 \mathrm{~min}$. The upper layer was removed again, and the tube was rinsed 3 times with sterile deionized water. Finally, samples were heated for $10 \mathrm{~min}$ at $90^{\circ} \mathrm{C}$ after adding sterilized water, and then the upper layer was collected from the tube.

\section{Genotyping of the Testes and Semen}

Of the microsatellite markers that are linked to the Hinai-dori breed, the $A B R 0633$ marker, which can distinguish the Hinai-dori breed from WL, was used to detect the Hinai-dori PGCs that were transferred into the WL embryos. The adjustment of reaction volume, PCR, and microsatellite genotyping were conducted using a procedure similar to that mentioned above.

\section{Progeny Test}

The manipulated male chickens were crossed with Hinai-dori females by artificial insemination and the plumage color of their offspring was examined. More than 100 hatchlings were produced per manipulated male chicken. For the manipulated male chicken that died during the progeny test, only the offspring produced before death were used for the progeny test. Blood was extracted from the underwing vein of the chicks identified as Hinai-dori by plumage color, and genotyping was conducted using the $A B R 0633$ marker in a similar method as mentioned above. When only the donor-derived Hinai-dori allele was detected in the offspring, it was identified as Hinai-dori; when both the Hinai-dori and WL alleles were detected, the offspring was not identified as Hinai-dori.

\section{Statistical Analyses}

The data for the chimera germline transmission rates were presented as the least square mean LSM(SEM), and were analyzed by one-way ANOVA using the general linear model (GLM) procedure of SAS (version 9.1.3; SAS Institute Inc., Cary, NC, USA). Differences in hatchability were evaluated using the chi-square test. Statistical significance was set at $P<0.05$.

\section{Results}

\section{Microsatellite Genotyping of Hinai-dori and WL}

The ABR0633 marker was linked to 1 allele in the Hinaidori breed. In contrast, WL introduced from NLBC had 2 alleles (271 and $273 \mathrm{bp}$ ), which differed from that of the Hinai-dori breed, and the frequencies of the 2 WL alleles were $78.9 \%$ and $21.1 \%$, respectively (Table 1 ). Therefore, in this study, we used these breeds as donor and recipient to produce germline chimeric chickens, and to verify the possibility of using the marker to identify germline chimeric chickens.

Table 1. Allele size and frequency of the $A B R 0633$ marker in Hinai-dori and White Leghorn

\begin{tabular}{cccc}
\hline \hline \multirow{2}{*}{ Size (bp) } & \multicolumn{2}{c}{ Allele frequency $(\%)$} \\
\cline { 3 - 4 } & & Hinai-dori & White Leghorn \\
\hline \multirow{3}{*}{ ABR0633 } & 261 & 100 & 0 \\
& 271 & 0 & 78.9 \\
& 273 & 0 & 21.1 \\
\hline
\end{tabular}


Table 2. Hatching and survival of recipient embryos produced by transfer of Hinai-dori PGCs into White Leghorn recipient embryos

\begin{tabular}{lccccc}
\hline \hline \multirow{2}{*}{ Transfer region } & No. of embryos & $\begin{array}{c}\text { No. of hatched chicks } \\
(\%)\end{array}$ & $\begin{array}{c}\text { No. of matured chicks } \\
(\%)\end{array}$ & \multicolumn{2}{c}{ Sex } \\
\hline Subgerminal cavity & 32 & 4 & 3 & Male & Female \\
Dorsal aorta & 5 & $\left(12.5^{\mathrm{b}}\right)$ & $\left(9.4^{\mathrm{b}}\right)$ & 2 & 1 \\
& & 4 & 4 & 2 & 2 \\
\hline
\end{tabular}

a,b Value with different superscripts in the same column are significantly different $(P<0.01)$.

Table 3. DNA analysis of testes and semen from the manipulated male chicks produced by transfer of Hinai-dori PGCs into White Leghorn recipient embryos

\begin{tabular}{|c|c|c|c|c|}
\hline \multirow[b]{2}{*}{ Transfer region/ID } & \multicolumn{2}{|c|}{ Testes } & \multicolumn{2}{|r|}{ Semen } \\
\hline & $\begin{array}{l}\text { Donor (Hinai-dori)- } \\
\text { derived allele }\end{array}$ & $\begin{array}{c}\text { Recipient (White Leghorn)- } \\
\text { derived alleles }\end{array}$ & $\begin{array}{l}\text { Donor (Hinai-dori)- } \\
\text { derived allele }\end{array}$ & $\begin{array}{c}\text { Recipient (White Leghorn)- } \\
\text { derived alleles }\end{array}$ \\
\hline \multicolumn{5}{|l|}{ Subgerminal cavity } \\
\hline D934 & - & + & - & + \\
\hline D935 & - & + & - & + \\
\hline \multicolumn{5}{|l|}{ Dorsal aorta } \\
\hline C963 & - & + & + & + \\
\hline C974 & - & + & + & + \\
\hline
\end{tabular}

Testes $=$ About $1 / 3$ of each testis was removed at 5 weeks of age.,$+=$ allele detectied,,$-=$ no allele detected.

The hatching rate of manipulated embryos and survival rate of manipulated chicks

PGCs obtained from embryonic gonads of the Hinaidori breed were microinjected into either the subgerminal cavity or dorsal aorta of WL embryos to produce germline chimeric chickens. The hatching rate of the manipulated embryos that were microinjected into the recipient subgerminal cavity $(12.5 \%$; 4/32) was significantly lower than that of the manipulated embryos that were microinjected into the recipient embryonic dorsal aorta $(80.0 \%$; $4 / 5$ ). Seventy-five percent of the chicks that hatched from those transferred into the subgerminal cavity matured, and two were males and one was female. In contrast, all chicks that hatched from those transferred into the dorsal aorta matured, and two were males and two were females (Table 2).

\section{Genotyping of the Testes and Semen}

The portions of both left and right testes that were removed from the 4 manipulated male chicks at 5-weeks old were used for genotyping to identify germline chimeric chickens. In all the testes of the manipulated male chicks (4/4) produced by the transfer of Hinai-dori PGCs into WL recipient embryos, only recipient-derived WL alleles were detected, and donor-derived Hinai-dori alleles were not detected (Table 3). Then the semen obtained from the matured manipulated male chickens was used for genotyping. Only recipient-derived WL alleles were detected from the semen of the two manipulated male chickens $(2 / 2)$ that were derived from the microinjection of Hinai-dori PGCs into the subgerminal cavity of WL embryos. In contrast, the donor-derived Hinai-dori allele was detected in the semen from the manipulated two male chickens (2/ 2) that were derived from the microinjection of Hinai-dori PGCs into the dorsal aorta of WL embryos (Table 3, Fig. 1).

\section{Progeny Test}

The manipulated male chickens were crossed with Hinai-dori females, and the plumage color and alleles of their offspring were examined to identify the germline transmission of donor Hinai-dori PGCs. No donor-derived offspring was obtained from the two manipulated male chickens $(2 / 2)$ produced by the transfer of Hinaidori PGCs into the subgerminal cavity. In contrast, donorderived offspring were obtained from the two manipulated chickens $(2 / 2)$ produced by the transfer of Hinai-dori PGCs into the embryonic dorsal (8.1\%; Table 4, Fig. 2). We confirmed that these two chickens that were produced by the transfer of Hinai-dori PGCs into the dorsal aorta of the WL recipient embryos are germline chimeric chickens both phenotypically and genotypically. Moreover, only the donor-derived Hinai-dori allele was detected in the genotyping of the blood from the offspring that were judged as the Hinai-dori breed by plumage color (Fig. 3). Therefore, we molecularly confirmed that these offspring were the Hinai-dori breed. 

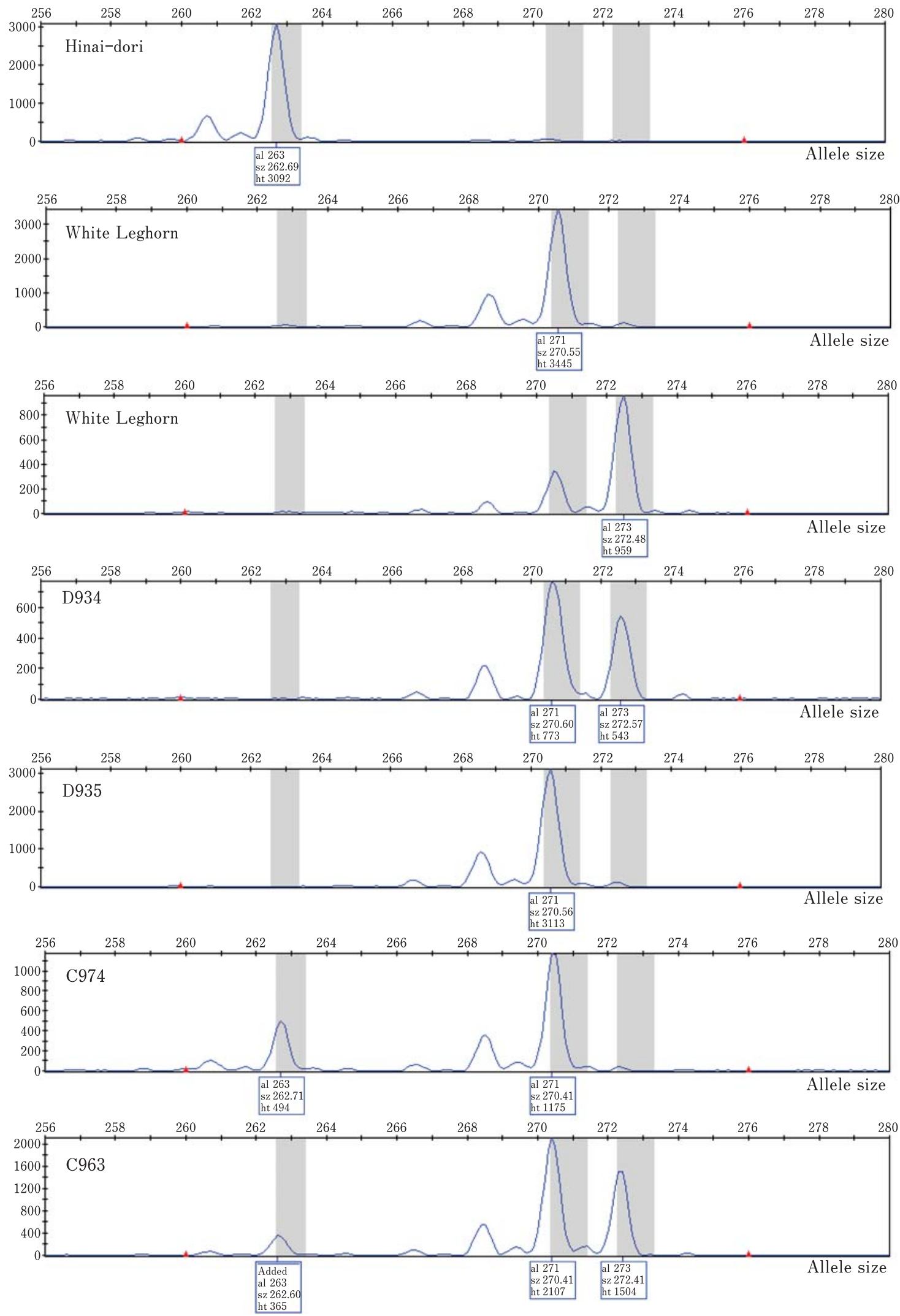

Fig. 1. Detection of germline chimerism using a Hinai-dori-specific marker in semen from manipulated male chicks that were produced by transfer of Hinai-dori PGCs. 263 bp: Hinai-dori allele; $271 \mathrm{bp}$ and $273 \mathrm{bp}$ : White Leghorn alleles. 
Table 4. Progeny test of the manipulated male chickens produced by transfer of Hinai-dori PGCs into White Leghorn recipient embryos

\begin{tabular}{lcccc}
\hline \hline Transfer region/ID & $\begin{array}{c}\text { No. of hatched } \\
\text { chicks }\end{array}$ & $\begin{array}{c}\text { Donor (Hinai-dori)- } \\
\text { derived offspring }\end{array}$ & $\begin{array}{c}\text { Analysis of DNA from offspring } \\
\text { Rate of donor- } \\
\text { derived offspring (\%) }\end{array}$ & $\begin{array}{c}\text { Donor (Hinai-dori)- } \\
\text { Recipient (White Leghorn)- } \\
\text { derived allele } \\
\text { derived alleles }\end{array}$ \\
\hline $\begin{array}{l}\text { Subgerminal cavity } \\
\text { D934 }\end{array}$ & 110 & 0 & 0 & NA \\
D935 & 69 & 0 & 0 & NA \\
LSM \pm SEM & & & 0 & NA \\
Dorsal aorta & 108 & & 1.9 & 14.3 \\
C963 & 7 & 2 & $8.1 \pm 6.2$ & - \\
C974* & & 1 & - \\
LSM \pm SEM & & & -
\end{tabular}

*, C974 died during the progeny test. NA, not applicable; +, allele detected; -, no allele detected.

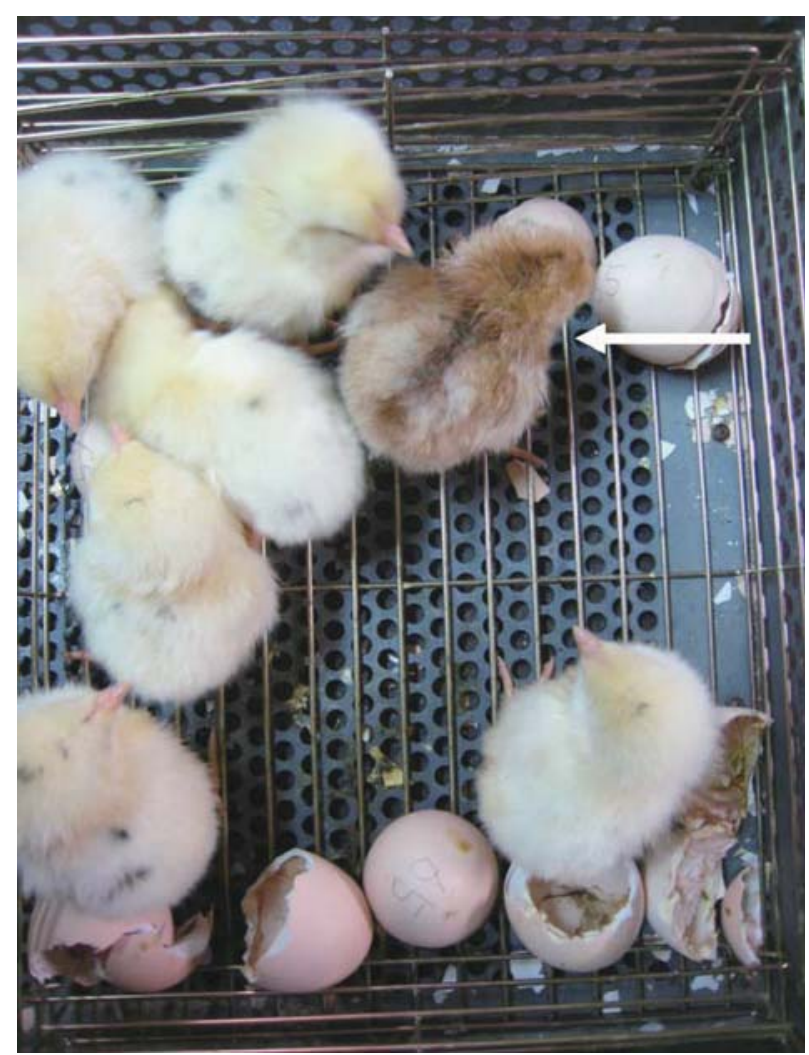

Fig. 2. Hatched offspring from the germline chimeric chickens. Arrow: Brown offspring are derived from donor Hinai-dori PGCs; White offspring with small patches are derived from recipient White Leghorn PGCs.

\section{Discussion}

Because of the technical difficulty of freezing embryos and transferring nuclei into ova in avian species, a unique reproductive technology based on producing germline chimeras by transfer of PGCs has been developed (Kagami,
2002; Tajima, 2002; Naito and Kuwana, 2004; Nakamura et al., 2009). Currently, testcross analysis using differences in plumage color between Dominant White (I), which is a characteristic of WL, and colored (i) chicken breeds is most widely used to identify germline chimeric chickens. However, this method is time consuming and laborious. Therefore, in this study, we verified the possibility of using a previously developed Hinai-dori-specific microsatellite marker (Rikimaru and Takahashi, 2007) to identify germline chimeric chickens among manipulated chickens produced by transfer of PGCs from Hinai-dori embryonic gonads.

To clarify whether the Hinai-dori-specific marker can be applied for the identification of germline chimerism, consistent results are required from both the molecular and testcross analyses. Since the Hinai-dori breed has colored plumage (i), it is possible to identify germline chimeric chickens by differences in the plumage color of offspring using WL with Dominant White (I) as the recipient embryos. The first step of this is to demonstrate whether the Hinai-dori-specific marker can distinguish the Hinai-dori breed from WL. Marker ABR0633, which is a Hinai-dori-specific microsatellite marker, was able to distinguish the Hinai-dori breed from WL that had been previously maintained at ALES (Rikimaru and Takahashi, 2007). However, that WL has not been maintained. Alternatively, WL introduced from NLBC has been maintained at ALES. Therefore, we demonstrated whether it is possible to distinguish the Hinai-dori breed from WL introduced from NLBC using the $A B R 0633$ marker. The results show that the marker was linked to 1 allele (263 bp) in the Hinai-dori breed, while WL had 2 different alleles ( $271 \mathrm{bp}$ and $273 \mathrm{bp}$ ). This result corresponded to that of Rikimaru and Takahashi (2007), who showed that it is possible to distinguish the Hinai-dori breed from WL . In this study, the Hinai-dori breed and WL introduced from NLBC were used as the donor and recipient breeds, respectively.

Gonadal PGCs, which distribute to the embryonic gon- 

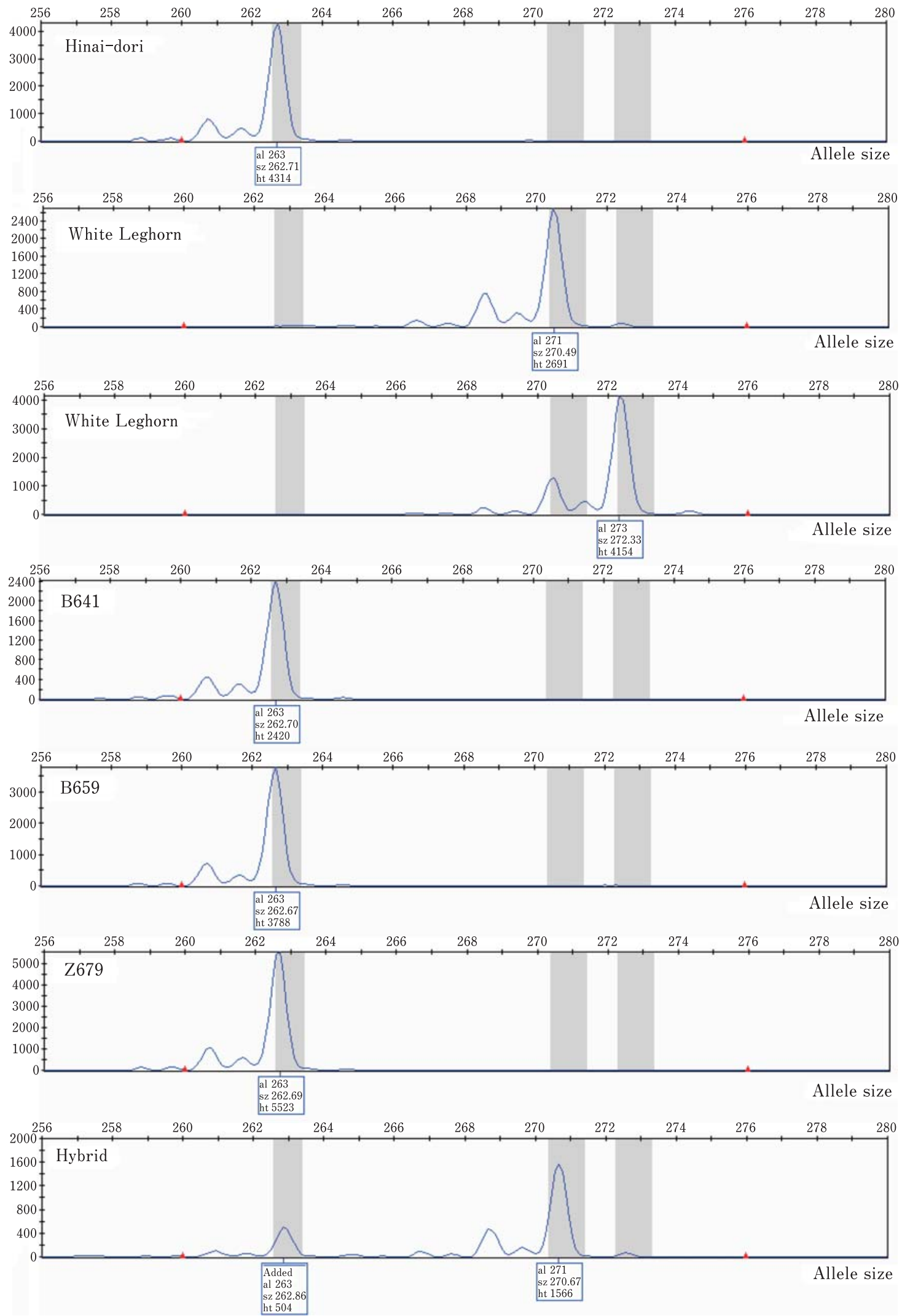

Fig. 3. Verification of offspring from the germline chimeric chickens. Verification was done by progeny testing the manipulated male chickens produced by transfer of Hinai-dori PGCs into White Leghorn recipient embryos and Hinai-dori females by using a Hinai-dori specific marker. B641, B659, Z679: Offspring judged as the Hinai-dori breed. 263 bp: Hinai-dori allele; 271 bp and 273 bp: White Leghorn alleles. 
ads, have been widely used for the production of germline chimeric chickens because many cells can be obtained from an embryo (Tajima et al., 1998; Park et al., 2003; Mozdziac et al., 2006). In this study, gPGCs obtained from the embryonic gonads of the Hinai-dori breed were microinjected into either the subgerminal cavity or the dorsal aorta of WL recipient embryos for germline-chimera production. Removal of the endogenous PGCs of recipient embryos prior to the transfer of donor PGCs should be an effective method for improving germline chimerism because the relative production of donor PGCs is increased. Therefore, in order to remove the endogenous PGCs, the recipient blastoderm was UV irradiated prior to the microinjection of donor PGCs into the subgerminal cavity. In contrast, prior to the microinjection of donor PGCs into the bloodstream through the dorsal aorta, a portion of the blood was removed from the recipient embryos. The hatching rate of the manipulated embryos in which the donor PGCs were microinjected into the subgerminal cavity was only $12.5 \%$. In contrast, the hatching rate of manipulated embryos in which the donor PGCs were microinjected into the embryonic dorsal aorta was $80.0 \%$, which was significantly higher than that of those microinjected into the subgerminal cavity. The reason for this difference is thought to be that the embryos from the donor PGCs that were microinjected into the subgerminal cavity were cultured ex vivo (Perry, 1988; Naito et al., 1990) while the embryos microinjected into the embryonic dorsal aorta were cultured in windowed eggshells. The ex vivo culture system leads to additional damage of the recipient embryo due to transfer of the embryo and its own yolk to a host eggshell. Therefore, hatchability in ex vivo culture is known to be lower than that in windowed eggshells, in which a small window is opened in the pointed end of the recipient eggshell. In addition to the difference in the culture method, UV irradiation also seemed to have a harmful influence on embryogenesis since UV irradiation causes extensive damage to cellular tissues (Reynaud, 1976).

Approximately 50-100 gPGCs collected from the gonads of Hinai-dori breed embryos, which were incubated for 6 days, were microinjected into the subgerminal cavity or dorsal aorta of WL recipient embryos. A progeny test showed germline transmission of donor PGCs in the manipulated rooster when the donor PGCs were transferred into bloodstream at stage 12-13. On the other hands, no donor PGC-derived offspring were obtained when the donor PGCs were transferred into the subgerminal cavity at stage X. Mozdziak et al. (2006) demonstrated that donor-derived offspring were obtained from germline chimeric chickens by transfer of a gPGC mass ( 560-2,000) into the subgerminal cavity, although the average frequency was only 5.3\%. The migration of chicken PGCs involves several steps: passive movement from the central zone of the area pellucida of the blastoderm to the germinal crescent region, entrance into the newly developing vascular system, temporal circulation in the bloodstream, and chemotactic migration to the future gonadal region (Nakamura et al., 2007). Taking these into consideration, the lack of germline transmission of donor PGCs that had been microinjected into the subgerminal cavity at stage $\mathrm{X}$ in this study would have been caused by low efficiency gonadal migration of donor PGCs due to the longer journey from the injected site to the recipient gonads. Therefore, the method whereby donor PGCs are transferred into the bloodstream of the recipient embryos is recommended for efficient production of germline chimeric chickens, because the distance and time spent migrating to the recipient gonads is the shortest among the currently available methods.

In the present study, offspring originating from Hinaidori PGCs were obtained from 2 germline roosters by transfer of donor PGCs into the bloodstream; however, the germline transmission rates were $1.9 \%$ and $14.3 \%$, which are still low. It is necessary improve the efficiency of obtaining donor-derived progeny from germline chimeric chickens in order to create better conditions for PGC-mediated genetic resource preservation in Hinaidori. The Lower frequency of donor-derived offspring in this study seems to be caused by insufficient removal of endogenous PGCs and inefficient spermatogenesis of female PGCs due to the introduction of mixed-sex PGCs. In the present study, we used the method whereby the embryonic blood is surgically removed in order to remove exogenous PGCs because this method is simple to perform. However, a noticeable enhancement in the germline transmission rate compared to that of the non-treated control chimeras could not be achieved using this method (23\% vs. 14\%; Naito et al., 1994b). Recently, a novel germline replacement technique that may further enhance the frequency of chimera production has been development. The efficient delivery of busulfan (1,4-butanediol dimethanesulfonate), an alkylating agent with cytotoxic effects on chicken germ cells, to chicken embryos (Nakamura et al., 2008) enables depletion of endogenous PGCs and repopulation with exogenous PGCs at an early developmental stage (Nakamura et al., 2009). Subsequently, these advances have been exploited for the production of chimeric chickens in which the recipient germline was replaced by donor cells with a 99.5\% transfer success (Nakamura et al., 2010b). This method will increase the frequency of obtaining donor-derived offspring from the chimeric chickens that are produced. To improve germline chimerism, the sex and fitness of the donor PGCs and recipient embryos are also important. The frequency of donor-derived offspring from germline chimeras was significantly higher for donor PGCs and recipient embryos of the same-sex compared with different-sex combinations (Naito et al., 1999). Tagami et al. (2007) reported that female PGCs in male gonads passed through the first and second meiotic divisions by adapting themselves to the male environment; however, their ability to complete spermatogenesis was limited.

The donor-derived Hinai-dori allele was detected in 
semen from the manipulated chickens that were phenotypically and genotypically identified as the Hinai-dori breed using a Hinai-dori-specific marker. However, the donor-derived Hinai-dori allele was not detected in the testes from these chickens at 5 weeks old. The presumed reason is that the amount of donor-derived DNA was lower than the limit of detection, because the proportion of germ cells in the testes is overwhelmingly low compared to the somatic cells in the testes, which are recipient derived. Although the testicular tissues were removed from the manipulated chickens at 5 weeks in this study, the donor-derived allele might have been detected if the testicular tissues were removed at earlier stages, such as just after hatching. Our results indicate that the Hinaidori-specific marker $A B R 0633$ is applicable for the identification of germline chimeric chickens by genotyping semen from the manipulated chickens; however, it is difficult to identify germline chimeric chickens by genotyping testes from the manipulated chicks at 5 weeks old.

Recently, molecular methods have been developed to identify germline chimeric chickens. Naito et al. (2004) verified the identification of germline chimeric chickens using an SNP in the D-loop region of mtDNA (Harumi et al., 2004) from WL, Barred Plymouth Rock (BPR), and Rhode Island Red. They succeeded in detecting donorderived BPR DNA from the gonads of 17-day-old recipient embryos that had BPR PGCs microinjected into the subgerminal cavity at stage $\mathrm{X}$. In the PMEL 17 gene, which plays an important role in the synthesis of melanosomes, 56 SNPs and 8 insertion/deletion polymorphisms have been reported (Kerje et al., 2004). Using a 9-bp insertion in exon 10 of PMEL 17, Choi et al. (2007) detected donor-derived DNA in the semen of germline chimeric chickens. However, using SNPs in the mtDNA D-loop region requires the selection of individual chickens with specific alleles prior to producing germline chimeric chickens, because the SNPs are polymorphic among individuals in a single breed. Utilization of the PMEL 17 gene is also restricted to using chicken breeds with Dominant white (I) as the donor or recipient for germline-chimera production. In contrast, the Hinai-dori-specific marker used in this study is linked to 1 allele in the Hinai-dori breed, and is able to distinguish the Hinai-dori breed from not only WL with Dominant white (I), but also other colored chicken breeds (Rikimaru and Takahashi, 2007). For this method, the breed of the recipient embryos is not restricted as in the other methods. Recent studies have shown that the migration capability to the gonads and/or proliferation capacity of the transferred exogenous PGCs differ among breeds (Nakamura et al., 2011), and the combination used as the donor and recipient greatly affects the transmission rates of the donor PGCs in the chimeric chickens (Naito et al., 1994b; Nakamura et al., 2010a). Our molecular identification method has a definite advantage in the selection of chicken breeds or lines used for recipients, because Hinai-dori-specific microsatellite markers are available for identifying Hinai-dori from other chicken breeds (Rikimaru and Takahashi, 2007).

The present study demonstrated the successful detection of the donor-derived Hinai-dori allele in the semen of germline chimeras that produced offspring from the Hinaidori PGCs that were identified both phenotypically and genotypically using the Hinai-dori-specific microsatellite marker ABR0633. We conclude that this Hinai-dorispecific microsatellite marker is suitable for the identification of germline chimeric chickens when the Hinai-dori breed is used for donor embryos, and, compared with testcrosses using differences in plumage color between the donor and recipient, the time and labor used for this method is reduced.

\section{References}

Choi JW, Lee EU, Shin JH, Zheng Y, Cho BW, Kim J, Kim H and Han JY. Identification of breed-specific DNA Polymorphisms for A simple and unambiguous screening system in germline chimeric chickens. Journal of Experimental Zoology, 307A: 241-248. 2007.

Science Council of Japan. 2006. Guidelines for Proper Conduct of Animal Experiments. Tokyo, Japan.

Eyal-Giladi H and Kochav S. From cleavage to primitive streak formation: A complementary normal table and anew look at the first stages of the development of the chick: I. General morphology. Developmental Biology, 49: 321-337. 1976.

Hamburger V and Hamilton HL. A series of normal stages in the development of the chick embryo. Journal of Morphology, 88: 49-92. 1951.

Han JY, Park TS, Hong YH, Jeong DK, Kim JN, Kim KD and Lim JM. Production of germline chimeras by transfer of chicken gonadal primordial germ cells maintained in vitro for an extended period. Theriogenology, 58: 1531-1539. 2002.

Harumi T, Sano A, Kagami H, Tagami T, Matsubara Y and Naito M. Polymerase chain reaction detection of single nucleotide polymorphisms in the chicken mitochondrial Dloop region. 2004. Animal Science Journal, 75: 503-507. 2004.

Hu XF, Xie B, Yu RS, Huang QZ, Zhang DF, Huang LS and Li Z. Generation of chicken germ-line chimeras by transferring PGCs and their identification by AFLP. Yi Chuan, 27: 367371.

Kagami H. Developmental genetic analysis of the avian primordial germ cells and the applications for production of chimeric chickens. Journal of Poultry Science, 39: 131-139. 2002.

Kerje S, Sharma P, Gunnarsson U, Kim H, Bagchi S, Fredriksson R, Schütz K, Jensen P, Heijne GV, Okimoto R and Andersson A. The Dominant white, Dun and Smoky color variants in chicken are associated with insertion/ deletion polymorphisms in the PMEL17 gene. Genetics, 168: 1507-1518. 2004.

Kuwana T, Kawashima T, Naito M, Yamashita H, Matsuzaki M and Takano T. Conservation of a threatened indigenous fowl (Kureko Dori) using the germline chimeras transplanted from primordial germ cells. Journal of Poultry Science, 43: 60-66. 2006.

Mozdziak PE, Wysocki R, Angerman-Stewart J, Pardue SL and 
Petitte JN. Production of chick germline chimeras from fluorescence-activated cell- sorted gonocytes. Poultry Science, 85: 1764-1768. 2006.

Naito $\mathbf{M}$ and Kuwana T. Production of chimeric chickens. Methods in Molecular Biology. Vol. 254.pp. 245-253. Humana Press Inc., New Jersey. 2004.

Naito M, Matsubara Y, Harumi T, Tagami T, Kagami H, Sakurai $\mathrm{M}$ and Kuwana T. Differentiation of donor primordial germ cells into functional gametes in the gonads of mixed-sex germline chimaeric chickens produced by transfer of primordial germ cells isolated from embryonic blood. Journal of Reproduction and Fertility, 117: 291-298. 1999.

Naito M, Nirasawa K and Oishi T. Development in culture of the chick embryo from fertilized ovum to hatching. Journal of Experimental Zoology, 254: 322-326. 1990.

Naito M, Sano A, Harumi T, Matsubara Y and Kuwana T. Migration of primordial germ cells isolated from embryonic blood into the gonads after transfer to stage $\mathrm{X}$ blastoderms and detection of germline chimaerism by PCR. British Poultry Science, 45: 762-768. 2004.

Naito M, Tajima A, Tagami A, Yasuda Y and Kuwana T. Preservation of chick primordial germ cells in liquid nitrogen and subsequent production of viable offspring. Journal of Reproduction and Fertility, 102: 321-325. 1994a.

Naito M, Tajima A, Yasuda Y and Kuwana T. Production of germline chimeric chickens, with high transmission rate of donor-derived gametes, produced by transfer of primordial germ cells. Morecular Reproduction and Development, 39: 153-161. 1994b.

Nakamura Y, Kagami $\mathrm{H}$ and Tagami T. Current states for regeneration of individuals via germline chimeras by transferring germ cells in animals. Journal of Animal Genetics, 37: 41-58. 2009. (in Japanese)

Nakamura Y, Usui F, Atsumi Y, Otomo A, Teshima A, Ono T, Takeda K, Nirasawa K, Kagami $\mathrm{H}$ and Tagami T. Effects of busulfan sustained-release emulsion on depletion and repopulation of primordial germ cells in early chicken embryos. Journal of Poultry Science, 46: 127-135: 2009.

Nakamura Y, Usui F, Miyahara D, Mori T, Ono T, Takeda K, Nirasawa K, Kagami $\mathrm{H}$ and Tagami T. Efficient system for preservation and regeneration of genetic resources in chicken: concurrent storage of primordial germ cells and live animals from early embryos of a rare indigenous fowl (Gifujidori). Reproduction, Fertility and Development, 22: 1237-1246. 2010a.

Nakamura Y, Usui F, Miyahara D, Mori T, Watanabe H, Ono T, Takeda K, Nirasawa K, Kagami H and Tagami T. Viability and functionality of primordial germ cells after freeze-thaw in chickens. Journal of Poultry science, 48: 57-63. 2011.

Nakamura Y, Usui F, Miyahara D, Ono T, Takeda K, Nirasawa $\mathrm{K}$, Kagami $\mathrm{H}$ and Tagami T. Germline reproduction by transfer of primordial germ cells into partially sterilized embryos in the chicken. Biology of Reproduction, 83: 130137. $2010 b$.

Nakamura Y, Yamamoto Y, Usui F, Atsumi Y, Ito Y, Ono T,
Takeda K, Nirasawa K, Kagami $\mathrm{H}$ and Tagami T. Increased production of donor primordial germ cells in chimeric gonads by sterilization of recipient embryos using busulfan sustained-release emulsion in chicken. Reproduction, Fertility and Development, 20: 900-907. 2008.

Nakamura Y, Yamamoto Y, Usui F, Mushika T, Ono T, Setioko AR, Takeda K, Nirasawa K, Kagami $\mathrm{H}$ and Tagami $\mathrm{T}$. Migration and proliferation of primordial germ cells in the early chicken embryo. Poultry Science, 86: 2182-2193. 2007.

Park TS, Jeong DK, Kim JN, Song GH, Hong YH, Lim JM and Han JY. Improved germline transmission in chicken chimeras produced by transplantation of gonadal primordial germ cells into recipient embryos. Biology of Reproduction, 68: 1657-1662. 2003. Poultry Science, 86: 2182-2193. 2007.

Perry MM. A complete culture system for the chick embryo. Nature, 331: 70-72. 1988.

Petitte JN, Clark ME, Liu G, Verrinder Gibbins AM and Etches RJ. Production of somatic and germline chimeras in the chicken by transfer of early blastodermal cells. Development, 108: 185-189. 1990.

Raynaud, G. The localization of primordial germ cells in Japanese quail embryos using a technic of ultraviolet irradiation. Comptes Rendus Hebdomadaires des Seances de l'Academie des Science, 282: 1195-1198. 1976.

Rikimaru K and Takahashi H. A method for discriminating a Japanese brand of chicken, the Hinai-jidori, using microsatellite markers. Poultry Science, 86: 1881-1886. 2007.

Rikimaru K and Takahashi H. A simple and efficient method for extraction of PCR-amplifiable DNA from chicken eggshells. Animal Science Journal, 80: 220-223. 2009.

Rikimaru K and Takahashi H. Verification of effectiveness of a method for identifying the Hinai-jidori chicken, by analyzing the product's form represented in the market. Japanese Journal of Poultry Science, 47: 1-7. 2010. (in Japanese)

Rikimaru K, Takahashi H and Nichols MA. An efficient method of early caponization in slow-growing meat-type chickens. Poultry Science, 90: 1852-1857. 2011.

Tagami T, Kagami H, Matsubara Y, Harumi T, Naito M, Takeda K, Hanada $\mathrm{H}$ and Nirasawa K. Differentiation of female primordial germ cells in the male testes of chicken (Gallus gallus domesticus). Molecular Reproduction and Development, 74: 68-75. 2007.

Tajima A. Production of germ-line chimeras and their application in domestic chicken. Avian and Poultry Biology Review, 13: 15-30. 2002.

Tajima A, Naito M, Yasuda Y and Kuwana T. Production of germ line chimera by transfer of primordial germ cells in the domestic chicken (Gallus domesticus). Theriogenology, 40: 509-519. 1993.

Tajima A, Naito M, Yasuda Y and Kuwana T. Production of germ-line chimeras by transfer of cryopreserved gonadal primordial germ cells (gPGCs) in chicken. Journal of Experimental Zoology, 280: 265-267. 1998. 